\title{
Phase-field Modeling with CALPHAD and CVM for Microstructural Evolution of Ni-base Superalloy
}

\author{
Jincheng Wang, ${ }^{1}$ Makoto Osawa, ${ }^{1}$ Tadaharu Yokokawa, ${ }^{1}$ Hiroshi Harada, ${ }^{1}$ Masato Enomoto ${ }^{2}$ \\ High Temperature Materials 21 Project, National Institute for Materials Science, \\ 1-2-1 Sengen, Tsukuba Science City, Ibaraki 305-0047, Japan \\ 2 Department of Materials Science, Ibaraki University, 4-12-1, Nakanarusawa, Hitachi 316-8511, Japan
}

Key words: Ni-Al, Phase-field Method, Microstructure Evolution, Rafting, Single Crystal

\begin{abstract}
To perform a quantitative simulation using the Phase Field Method (PFM) in a real alloy system, a new strategy of modeling the microstructure evolution of Ni-base superalloy is proposed in the present paper. In this strategy, the Calculation Phase Diagram (CALPHAD) method and Cluster Variation Method (CVM) are combined with the PFM, in which the four sub-lattice model is used to evaluate the free energy density, while CVM is used to calculate some parameters, such as the lattice misfit and gradient coefficients in phase-field equations. The microstructure evolution of a Ni-Al binary alloy at the temperature of $1000 \mathrm{~K}$ has been simulated; the elastic energy due to the lattice misfit and elastic inhomogeneity between $\gamma$ and $\gamma^{\prime}$ phases have also been taken into account. The simulated microstructures are formed by cuboidal coherent $\gamma^{\prime}$ precipitates with a strong alignment in a certain direction, and all the precipitates are single-domain particles. The influence of the volume fraction of the $\gamma^{\prime}$ phase on the final microstructures is also investigated. Results show that with the increasing of the volume fraction, the size distribution of the precipitates becomes broader and the aspect ratio of the cuboidal coherent precipitates becomes smaller, which may result from the interactions between the precipitates that become stronger because of the high-volume fraction. Moreover, the directional coarsening phenomenon of Ni-Al alloy with 18at. \%Al has been reoccurred with this strategy when the various external stress conditions are applied, which shows the same conclusion as Pineau's directional coarsening map.
\end{abstract}

\section{Introduction}

As one of the most important classes of high-temperature structural materials, Ni-base superalloy exhibits a truly unique combination of properties at elevated temperatures. The microstructures in a Ni-base superalloy typically are a two-phase mixture of $\gamma$ and $\gamma^{\prime}$ phases, which are the disordered $f c c$ phase (Ni solid solution) and the ordered $\mathrm{L}_{2}$ phase $\left(\mathrm{Ni}_{3} \mathrm{Al}\right.$ intermetallic compound), respectively. Precipitation hardening by the ordered phase is the major strengthening mechanism for Ni-base superalloys, which makes the microstructure control of $\gamma$ phase and $\gamma^{\prime}$ phase the key technique in the development of advanced Ni-base superalloys. However, since the factors that influence the microstructure formation are extensive, which increase the difficulty and complexity of controlling the microstructure, many trial-and-error experiments are often necessary when searching for the best combination of desired microstructures and material properties. One obvious choice for overcoming this shortcoming is to use simulation for modeling the microstructural evolution.

The Phase-Field Method (PFM) is a newly developed and very powerful tool for the simulation and prediction of complex microstructure evolution. This has been demonstrated in a lot of areas, such as dendrite growth, spinodal decomposition, grain growth and so on. As to the order-disorder transformation, especially for the $\gamma-\gamma^{\prime}$ in the Ni-base superalloy, the PFM has numerous advantages, as it ensures that many factors are taken into account, such as the coherency strain, elastic anisotropy, external stress, and $\mathrm{L}_{2}$ ordering of the precipitate phase. These advantages make it very convenient to study the coarsening behavior of the precipitates in a Ni-base superalloy. Therefore, many studies have been done for predicting the microstructure evolution of Ni-base superalloys with the phase field method ever since the first phase field model for precipitation coarsening was proposed [1-8].

However, although tremendous success in this area has been achieved, additional progress is required in order to apply this method in a real alloy system. The simulation with real parameters is highly anticipated by materials scientists. Quantitative simulation by coupling real thermodynamic databases and kinetic databases with PFM has recently been receiving more and more attention [6-8]. There are two typical ways to couple a thermodynamic or kinetic database with the phase field method. One is to use a thermodynamic model to directly calculate the free chemical energy, such as that used by J. Zhu et al. [6], who coupled the sub-lattice model with the PFM to study the precipitate shape evolution in a Ni-base superalloy. However, in PFM, the free energy form for different phases should be a uniform style; as a result, only a few thermodynamic models (such as the sub-lattice model) can be used directly in this way. Another way is to combine PFM with some thermodynamic software (such as Thermo-calc) so that the free energy and some kinetic coefficient can be determined through a user-programming interface. U. Grafe et al. [7] have used such a method to study the Ostwald-ripening behavior of a Ni-base by accessing Thermo-calc through its user-programming interface. In addition, Q. Chen et $a l$. [8] performed a quantitative study modeling precipitate growth and dissolution with inputs from a CALPHAD thermodynamic database and a DICTRA kinetic database. Success has been achieved with both methods; however, there does still exist some room for improvement with quantitative modeling. There are some parameters that are difficult to obtain regardless of whether 
thermodynamic or kinetic databases are used in the experiments. They include the lattice misfit between the order phase and the disorder phase and the gradient coefficients of interface energy. These parameters are very important for the simulation but not easily measured in experiments. It is difficult to obtain highly accurate results, especially, the gradient coefficients of the energy. Fortunately, the Cluster Variation Method (CVM) may provide a solution. CVM, one of the most reliable tools used to provide detailed information of the atomic arrangements of a given alloy, can be used to calculate not only the free-energy variations with long-range or short-range order parameters, the equilibrium profile of composition and order parameters, but also some of the parameters that are needed in the PFM simulations, such as the lattice misfit and the interface energy, with high accuracy. Therefore, in order to perform a quantitative simulation, in the present paper, a new strategy of quantitative simulation is proposed, which combines PFM, CALPHAD, and CVM, to study the dynamics and morphological evolution of $\gamma$ and $\gamma^{\prime}$ phases in a real Ni-Al binary alloy system.

\section{Model}

\subsection{Phase field model}

In the present study, the phase field model proposed by Y. Wang et al. [1] is adopted. In the model, the composition field and three long-range order (LRO) parameter fields have been used to describe the concentration profile and the $\mathrm{Ll}_{2}$ order phase structure. The spatial-temporal evolution of the LRO parameters can be obtained by solving the time-dependent Ginzburg-Landau equations, while the temporal evolution of composition field can be described by the non-linear Cahn-Hilliard diffusion equation, which are:

$$
\begin{aligned}
& \frac{\partial \eta_{i}}{\partial t}=-L \frac{\delta F}{\delta \eta_{i}}, i=1,2,3, \\
& \frac{\partial c}{\partial t}=M \nabla^{2} \frac{\delta F}{\delta c}
\end{aligned}
$$

where $\mathrm{L}$ and $\mathrm{M}$ are the structural relaxation and diffusion mobility, c, the composition filed, $\eta_{\mathrm{i}}$, the order parameter field, which can describe the four different domains in the $\mathrm{L} 1_{2}$ phase structure, and $\mathrm{F}$, the total free energy of the system, which includes the chemical free energy, interface energy, and elastic energy, i.e., $F=F_{\text {chemical }}+F_{\text {elastic }}$. In $F_{\text {chemical }}$, the chemical free energy and interface energy are included, which can be introduced through the gradients of the field variables in the diffuse interface phase-field model. According to the Cahn-Hilliard diffuse interface energy model [9], the total chemical free energy (interface energy is included) can be written as:

$$
F_{\text {chemical }}=\int_{V}\left(f\left(c, \eta_{1}, \eta_{2}, \eta_{3}\right)+\frac{\alpha}{2}(\nabla c)^{2}+\sum_{i=1}^{3} \frac{\beta_{i}}{2}\left(\nabla \eta_{i}\right)^{2}\right) d V
$$

where $\alpha$ and $\beta_{\mathrm{i}}$ are gradient energy coefficients, which can be determined from the total interface energy, and $f\left(c, \eta_{1}, \eta_{2}, \eta_{3}\right)$ is the Helmholtz free energy density. The composition-dependent elastic strain energy $F_{\text {elastic }}$ due to elastic inhomogeneity can be solved with the method of high-order approximation proposed by Hu et al. [10].

\subsection{Free energy calculated with the sub-lattice model}

To obtain reasonable simulation results with PFM, the free energy calculation should be highly emphasized because the free energy of the system, as a function of some order parameters, will influence the driving force and path of the microstructural evolution. Currently, in most cases, the chemical free energy in PFM is described as a Landau-type polynomial form [1-5], in which most parameters have no physical meanings and have to be determined empirically through trial-and-error. Moreover, this Landau-type polynomial is not likely to obtain a very accurate description of the real energy variation of the system. Therefore, in order to apply the phase-field method to a real alloy system, the local chemical free energy as a function of field variables can be constructed using the CALPHAD method. Sub-lattice model is a very successful thermodynamic model, especially for the orderdisorder transition. The most important point in a sub-lattice model is that the free chemical energy is a uniform form for both the order and disorder phases, which can ensure continuous variation in the interface area, while a uniform form energy formulation is the main feature of phase field method and also a necessary condition for the energy function. This means that the sub-lattice model is very suitable to PFM. In the present paper, a four sub-lattice thermodynamic model was used to calculate the local chemical free energy the system. Using the order parameters in the phase field model to replace the site occupancies possibilities of $\mathrm{Al}$ atoms on the sub-lattices, the free chemical energy can be described as:

$$
\begin{aligned}
G\left(c, \eta_{i}\right) & =c G_{0}^{A l}+(1-c) G_{0}^{N i}+c(1-c) L_{0}+L_{1}(2 c-1) \\
& +L_{2}(2 c-1)^{2}+L_{3}(2 c-1)^{3}+4 U_{1} c^{2} \sum_{i=1}^{3} \eta_{i}^{2} \\
& 12 U_{4}(1-2 c) c^{2} \sum \eta_{i}^{2}-48 U_{4} c^{3} \eta_{1} \eta_{2} \eta_{3}+R T / 4 \\
& \left\{\left[c\left(1+\eta_{1}+\eta_{2}+\eta_{3}\right)\right] \ln \left[c\left(1+\eta_{1}+\eta_{2}+\eta_{3}\right)\right]+\right. \\
& {\left[1-c\left(1+\eta_{1}+\eta_{2}+\eta_{3}\right)\right] \ln \left[1-c\left(1+\eta_{1}+\eta_{2}+\eta_{3}\right)\right] } \\
& +\left[c\left(1-\eta_{1}-\eta_{2}+\eta_{3}\right)\right] \ln \left[c\left(1-\eta_{1}-\eta_{2}+\eta_{3}\right)\right]+ \\
& {\left[1-c\left(1-\eta_{1}-\eta_{2}+\eta_{3}\right)\right] \ln \left[1-c\left(1-\eta_{1}-\eta_{2}+\eta_{3}\right)\right] } \\
& +\left[c\left(1-\eta_{1}+\eta_{2}-\eta_{3}\right)\right] \ln \left[c\left(1-\eta_{1}+\eta_{2}-\eta_{3}\right)\right]+ \\
& {\left[1-c\left(1-\eta_{1}+\eta_{2}-\eta_{3}\right)\right] \ln \left[1-c\left(1-\eta_{1}+\eta_{2}-\eta_{3}\right)\right] } \\
& +\left[c\left(1+\eta_{1}-\eta_{2}-\eta_{3}\right)\right] \ln \left[c\left(1+\eta_{1}-\eta_{2}-\eta_{3}\right)\right]+ \\
& {\left.\left[1-c\left(1+\eta_{1}-\eta_{2}-\eta_{3}\right)\right] \ln \left[1-c\left(1+\eta_{1}-\eta_{2}-\eta_{3}\right)\right]\right\} }
\end{aligned}
$$

where $G_{0}^{A l}$ and $G_{0}^{N i}$ are the Gibbs energy of pure $\mathrm{Al}$ and $\mathrm{Ni}$, respectively, $\mathrm{L}_{0}, \mathrm{~L}_{1}, \mathrm{~L}_{2}$, and $\mathrm{L}_{3}$, interaction parameters for excess energy, and $U_{1}$ and $U_{4}$, parameters for bond energy. All of these parameters can be found in the thermodynamic database [11]

According to Eq. (4) and setting the three order parameters $\left(\eta_{i}, i=1,2,3\right)$ equal to each other, the contour of the free energy is plotted as shown in Fig.1. It can be concluded that there are two wells in the surface and that they correspond to the equilibrium 


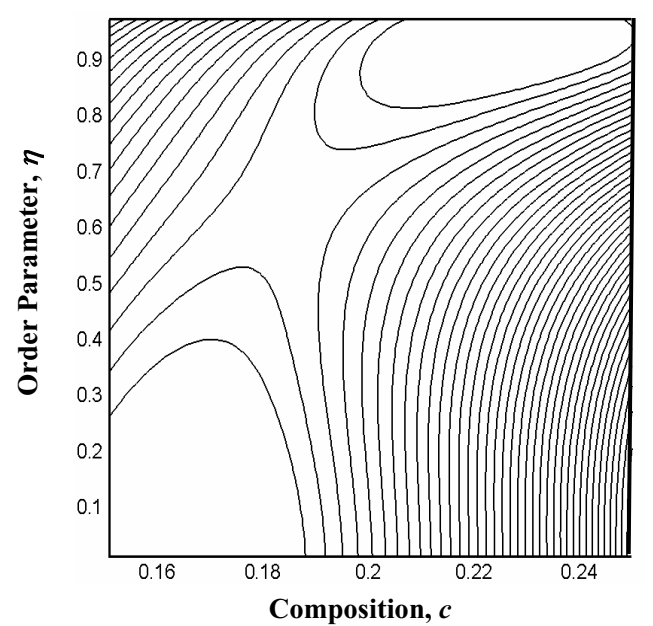

Fig.1 Contour plot of the free energy surface given by Eq. (4) on the $c-\eta$ plane

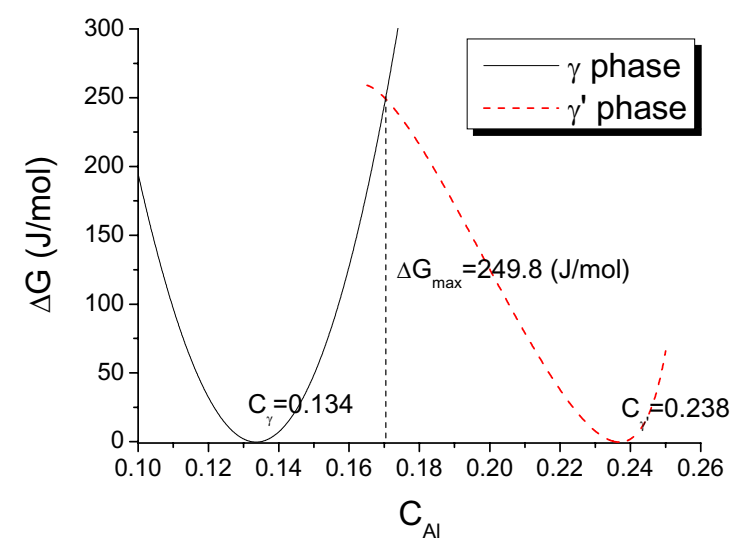

Fig. 2 Chemical free energy of the $\gamma$ and $\gamma^{\prime}$ phases of Ni-Al alloy calculated by the four sub-lattice model at $1000 \mathrm{~K}$

states of the disorder and order phases. The values of the composition and the order parameter for these two phases are $c=0.134, \eta=0$ and $c=0.238, \eta=0.95$, respectively.

Figure 2 shows the chemical free energy of $\gamma$ phase and $\gamma^{\prime}$ phase of the Ni-Al alloy as a function of composition calculated by the four sub-lattice model at the temperature of $1000 \mathrm{~K}$. From the figure, it can be determined that the equilibrium composition of $\gamma$ phase and $\gamma^{\prime}$ phase at the stress-free state are 0.134 and 0.238 , respectively, and the maximum driving force $\Delta G_{\max }$ is approximately $249.8 \mathrm{~J} / \mathrm{mol}$. It should be noted that the function of $f\left(c, \eta_{1}, \eta_{2}, \eta_{3}\right)$ in Eq. (3) is the free energy density, while, in Eq.(4), $G\left(c, \eta_{1}, \eta_{2}, \eta_{3}\right)$ is the free energy with the relation of $f=G / V_{m}$, where $V_{m}$ means the mole volume.

\subsection{Cluster Variation Method (CVM)}

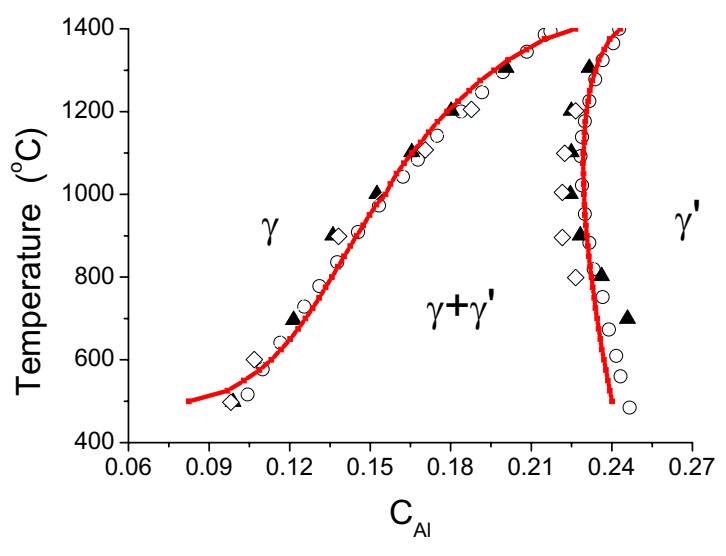

Fig.3 Phase boundary of $\gamma$ and $\gamma^{\prime}$ calculated by CVM and comparisons of some experimental results. $\mathbf{C}, \mathrm{CVM}, \circ$, Ansara et al [16], updated by Dupin [16], $\mathbf{\Lambda}$, Jia [16], and $\diamond$, Watanabe et al [16].

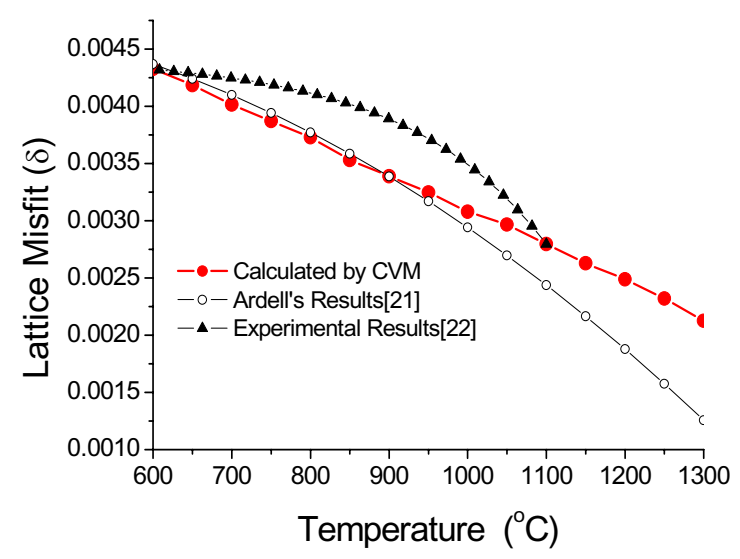

Fig.4 Lattice misfit between $\gamma$ and $\gamma^{\prime}$ phases calculated by CVM and compared with experimental results

In the present study, the tetrahedron approximation and the phenomenological Lennard-Jones pair potential have been used in the CVM, which have been shown to be powerful in the calculation of the phase boundary and lattice parameters of a Nibase superalloy [12]. R. Kikuchi et al. [13] have also used the CVM to calculate the inter-phase boundary (IPB) and the antiphase boundary (APB) energies with multi-atom interactions, in which a super cell with many layers was used. Recently, M. Asta et al. $[14,15]$ used a CVM to predict the interface properties of Al$\mathrm{Li}$ and $\mathrm{Al}-\mathrm{Ag}$ alloys, which demonstrated that a CVM could be used to calculate the interface energy. Therefore, in this study, a CVM was used to calculate the lattice parameters and the interphase boundary (IPB) energy between order and disorder phases of the Ni-Al binary alloy system.

To verify the accuracy of the CVM simulation, we calculated the phase boundary between the order phase and the disorder 


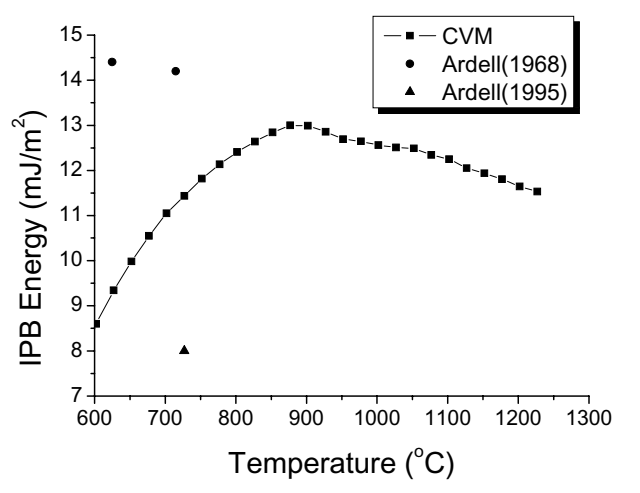

Fig.5 Calculated interface energy for (100) IPB by CVM and comparisons of some other results

phase. Figure 3 shows the phase boundary of $\gamma$ and $\gamma^{\prime}$ in the Ni-Al alloy system calculated by CVM and a comparison with some experimental results. The CVM results have very good agreement with the experiments (all experimental results come from reference 16). Moreover, the equilibrium composition predicted by CVM ( $c=0.13$ and $c=0.230$ for the disorder and order phases at the temperature of $1000 \mathrm{~K}$ ) is in accord with that predicted by the sub-lattice model, as shown in Fig.2. Therefore, it can be concluded that the CVM is a very reliable method.

Figure 4 shows the variation of the lattice misfit $(\delta)$ between $\gamma$ phase and $\gamma^{\prime}$ phase calculated with the temperature and comparisons with some experimental results, in which $\delta=\left(\delta_{\gamma^{\prime}}-\delta_{\gamma}\right) / \delta_{\gamma^{\prime}} . \delta_{\gamma}$ and $\delta_{\gamma^{\prime}}$ are the lattice parameters for the disorder phase and the order phase, respectively, which are determined by the CVM. In conclusion, the simulation and the experimental results were in good agreement. It should be noted that, in the figure, the experimental results [17] are measured in the case of a semi-coherent state, and Ardell's results are the formula given in his paper [18].

In Fig.5, the inter-phase boundary (IPB) energy of the (100) plane between the order phase and the disorder phase is also calculated by CVM. It shows, in the temperature range considered, that the interface energy first increases with temperature and then decreases. The value of the interface energy is about $10 \mathrm{~mJ} / \mathrm{m}^{2}$, which agrees with Ardell's values $[19,20]$. From this interface energy, the energy gradient coefficient can be determined. Therefore, using CVM, the parameters in phase field models can be determined with high accuracy. After the interface energy is determined, the gradient coefficients of the interface energy can be obtained. The method used in the present study is essentially the same as that used by Asta and Hoyt [14].

According to the description above, the strategy for a quantitative simulation of the phase field method can be established: it requires the use of the CALPHAD method (four sub-lattice model) to calculate the free energy of the system and the CVM to calculate the lattice misfit $\delta$ and energy gradient coefficients, $\alpha$ and $\beta_{\mathrm{i}}$. In addition, the kinetic coefficients (M and L) for the two field variables, which describe the mobility, can be calculated from the kinetic database at a given temperature.
Therefore, coupling the CALPHAD and CVM with PFM, all the parameters are determined and the phase-field equations can be solved numerically. Under this strategy, the simulation of microstructure evolution in the $\mathrm{Ni}-\mathrm{Al}$ binary alloy has been performed.

\section{Results and Discussion}

\subsection{Parameters and conditions}

The simulation is on a two-dimensional square domain using a uniform lattice of $256 \times 256$ with periodic boundary conditions. The initial condition of the system is just quenching at the temperature of $1000 \mathrm{~K}$. The elastic constants at that temperature of $\gamma^{\prime}$ phase and $\gamma$ phases are $C_{11}^{\gamma^{\prime}}=245 \mathrm{GPa}$, $C_{12}^{\gamma^{\prime}}=145 \mathrm{GPa} \quad, \quad C_{44}^{\gamma^{\prime}}=96 \mathrm{GPa} \quad$ and $C_{11}^{\gamma}=202 \mathrm{GPa}$, $C_{12}^{\gamma}=135 \mathrm{GPa}, C_{44}^{\gamma}=103 \mathrm{GPa}$, respectively [23], and the lattice misfit calculated by CVM is 0.004 . The interface energy at this temperature is about $11.5 \mathrm{~mJ} / \mathrm{m}^{2}$, from which the gradient coefficients can be determined, $\alpha=2.5 \times 10^{-9} \mathrm{~J} / \mathrm{m}$ and $\beta_{i}=6.0 \times 10^{-12} \mathrm{~J} / \mathrm{m}$. The time step used in the simulation is $\Delta \mathrm{t}=0.005$. The numerical solutions of the phase field equations were obtained using the semi-implicit Fourier-spectral method $[21,22]$, which has been proved to be an efficient numerical method for phase field equations. At the nucleation stage, the Langevin noise term, which are taken to be Gaussian distributed and satisfy the fluctuation-dissipation theorem, is added to the phase field equations to aid the nucleation by overcoming the nucleation barrier. After enough nuclei have formed, the noise terms are removed.

\subsection{Comparisons of calculated profile of composition and order parameter by PFM and CVM}

As mentioned above, the CVM can be used to obtain highly accurate calculations of the equilibrium profile of the composition and order parameters. Therefore, first of all, we will compare the equilibrium profiles of the composition and the order parameter near the diffuse interface calculated from the PFM and CVM. Figure 6 shows the composition profile of Al, and Fig.7 is the order parameter profile, both of which show good agreement with each other. The equilibrium composition of the disorder phase is 0.134 and 0.136 for the PFM and CVM, respectively. In the case of the order phase, the values are 0.230 and 0.235 , respectively. The equilibrium order parameters for both phases are $0,0.99$ for the CVM and 0, 0.95 for the PFM. From that it can be concluded that the phase field method under the strategy of coupling the thermodynamic model can predict the proper equilibrium value of the composition and order parameters. In the CVM simulation, it also verified that the interface between the order and disorder phases has a certain thickness, which means, at the equilibrium state, the interface is diffuse and not a sharp one. Moreover, in experimental aspect, H. Harada et al. [24] also have proved such kind of diffuse interface character between $\gamma^{\prime}$ and $\gamma$ phases using a Filed Ion Microscope (FIM) technique. They concluded that the change in the composition between the bulk $\gamma^{\prime}$ and $\gamma$ phases is not sudden but occurs over a distance of some 10 atomic layers. Moreover, the thickness of the diffuse interface in the phase field 


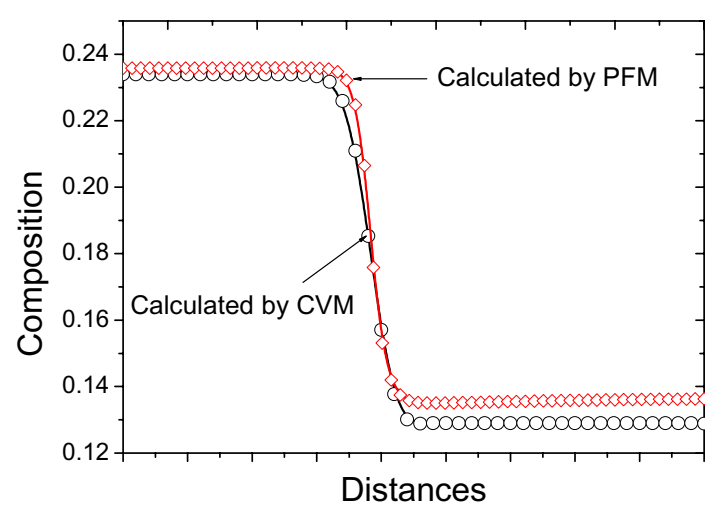

Fig.6 The comparisons of composition profiles calculated by PFM and CVM

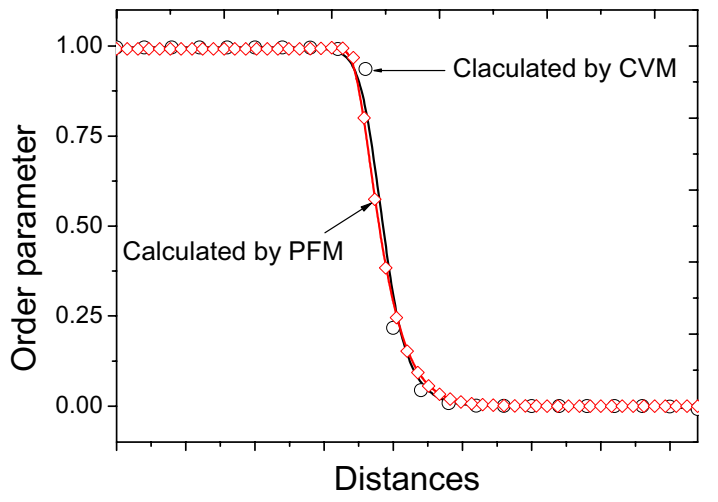

Fig.7 The comparisons of order parameter profiles calculated by PFM and CVM
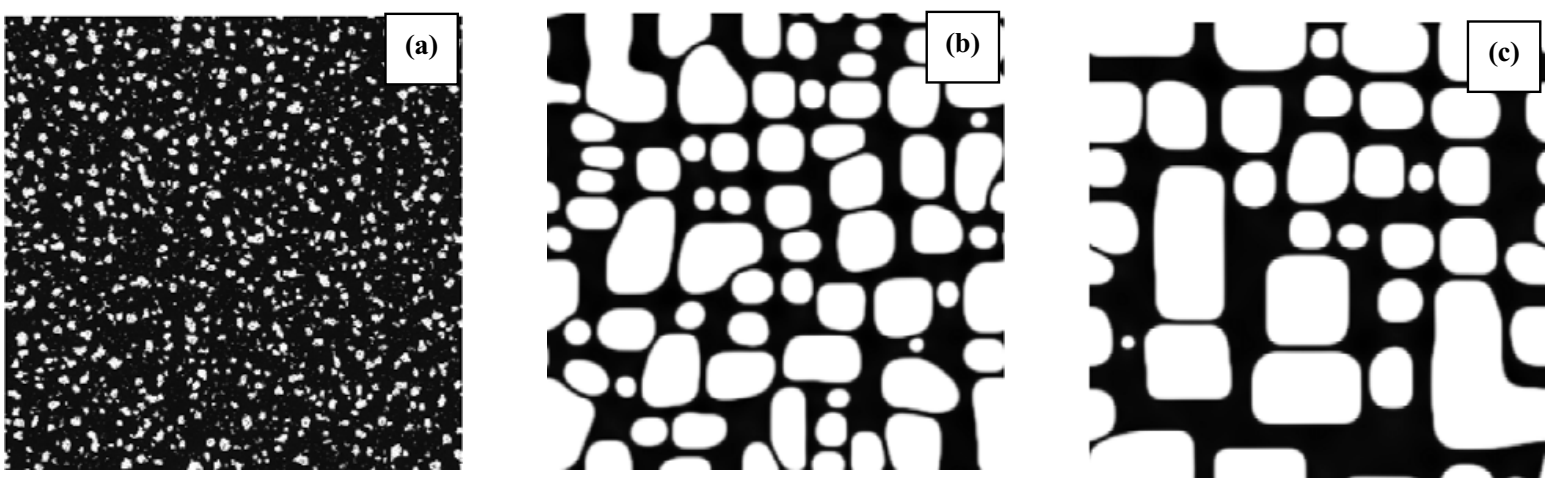

Fig.8 Simulated Microstructures of Ni-18at.\%Al alloy at 1000K at different reduced times.

(a) $t^{*}=6$, (b) $t^{*}=500$, (c) $t^{*}=2000$
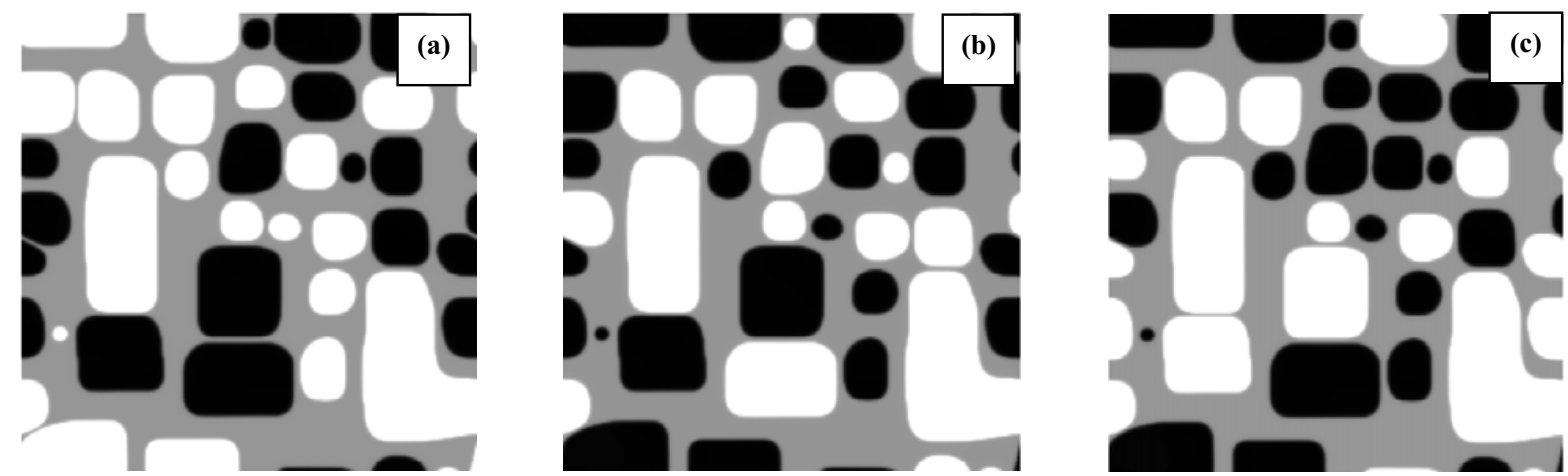

Fig.9 The order parameters field of Ni-18at. \%Al alloy at $1000 \mathrm{~K}$ when the reduced simulation time is 2000

(a) $\eta_{1}$

simulation is about $1.2 \mathrm{~nm}$, which is quite close to the values predicted by CVM at $1000 \mathrm{~K}$ (about $\sim 1.5 \mathrm{~nm}$ ) and the experimental results [24] (some 10 atomic layers, about $\sim 1.5 \mathrm{~nm}$ ). Therefore, the diffuse interface model, which has been adopted in the phase (b) $\eta_{2}, \quad$ (c) $\eta_{3}$

field method and also is one of the most important features of the PFM, is quite reasonable.

3.3 Microstructure evolution of Ni-18at $\% \mathrm{Al}$ at $1000 \mathrm{~K}$ 
Figure 8 shows the simulated microstructures of $\mathrm{Ni}-18 \mathrm{at} \% \mathrm{Al}$ at $1000 \mathrm{~K}$ at different evolution times, in which shades of gray represent the value of Al composition, e.g., the higher the value, the brighter the shade. Figure 8 (a) shows the microstructure at the point right after the nucleation stage has finished, when the reduced simulation time $t^{*}$ equals 6 , in which thousands of spherical nuclei have formed. Figure 8 (b) and (c) demonstrate the structure obtained by the growth and coarsening of the initial structure (Fig. 8 (a)) consisting of randomly distributed spherical precipitates when the reduced simulation times are $t^{*}=500$ and $\mathrm{t}^{*}=2000$, respectively. In Fig.9, the final order parameter fields are shown. The black and white ones stand for the two equilibrium values of order parameters, and the gray one is the equilibrium value of the disorder phase. Using these order parameters, the four different domains of the order phase can be distinguished, which are $\eta_{\max }(1,1,1), \eta_{\max }(-1,-1,1), \eta_{\max }(1,-1,-$ $1)$, and $\eta_{\max }(-1,1,-1)\left(\eta_{\max }=\eta_{1 \max }=\eta_{2 \max }=\eta_{3 \max }\right)$.

From Figs. 8 and 9, it can be seen that, although there exist four different domain precipitates, all the precipitates are singledomain particles. This means that no anti-phase boundary (APB) is formed in the ordered phase. Usually, the APB energy is prohibitively high, generally, twice as large as the interphase boundary (IPB) energy. Therefore, the APB is very unstable; if it exists, it will change into two IPBs by splitting the particle into two single-domain precipitates to arrive at a more stable state. The existence of different domains in the microstructures may also have a specific effect on the formation of the final structure. It is very interesting that the final microstructure is a cuboidal structure with a certain direction alignment. The elastic inhomogeneity between the order and disorder phases may produce, for hard precipitates, a structure that tends to surround a soft matrix to minimize the elastic energy. On the other hand, the cuboidal structures with the feature of alignment in a Ni-base superalloy may not come solely from the elastic inhomogeneity between $\gamma$ phase and $\gamma^{\prime}$ phase because the experimental results show that the elastic inhomogeneity in a Ni-base superalloy is small (the ratio anisotropy $\left(\delta=2 C_{44} /\left(C_{11}-C_{12}\right)\right.$ ) of $\gamma$ and $\gamma^{\prime}$ are 1.92 and 3.08, respectively). Therefore, these microstructures may form as a result of the different domains that exist within the

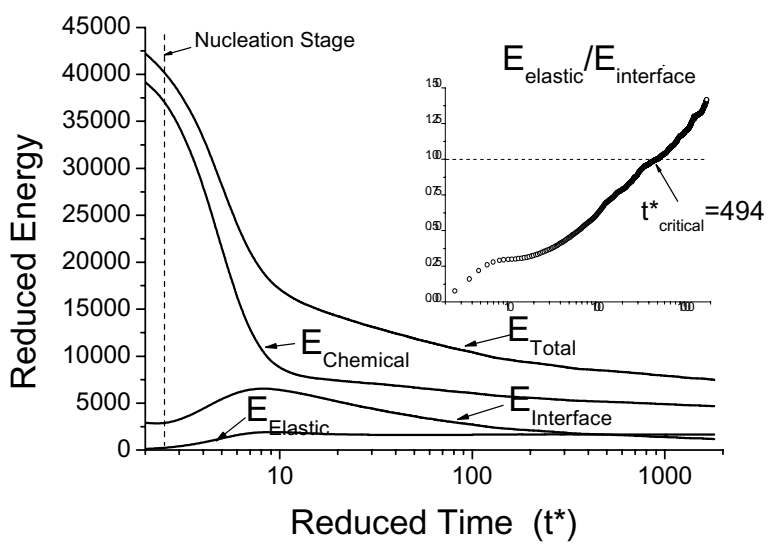

Fig.10 The different energy variations with reduced time correspond to the evolution process as shown in fig. 8 structures.

The coalescence of the precipitates may come from two processes. One is that different precipitates, which are very near, will combine into one big precipitate through a strain-induced uphill diffusion. However, because the domain of the precipitates may not be the same, this combination will not occur in the case of different domains. As a result, the near precipitates for one target will not be in the same domain as the target in the final structures, as shown in Fig.9. Another process is the conventional Ostwald-ripening, where small particles shrink due to emission while large particles grow by absorption. The driving force for this process comes from the interface energy minimization of the system.

In general, the microstructure evolution is controlled by the total energy of the system. Figure 10 shows the energy variation with the simulation time. For the system, according to the principle of energy minimization, it will evolve to the direction of low energy. Therefore, the total energy of the system decreases with the evolution process except in the nucleation stage (because, in the nucleation stage, the system needs external energy input to overcome the nucleation barrier). The total energy of the system includes three parts, namely, chemical free energy, interface energy, and elastic energy. As shown in Fig.10, the chemical energy also decreases monotonically with the evolution process (except in the nucleation stage). However, the interface energy first increases and then decreases. At first, the growth of the precipitates is the main process. Therefore, the surface of the particles increases, inducing an increase in the interface energy. When the evolution process gets to a certain period, the growth process is not the main part. This is because the Ostwald-ripening process, resulting in a decrease in the interface energy, has exceeded the growth process. The elastic energy of the system is just slowly increasing, which is the result of the volume fraction of precipitates increasing and the elastic inhomogeneity increasing The critical point of the interface energy equal to the elastic energy has also been plotted in Fig.11. It may be concluded that, below this critical point, the interface energy has much more influence on the system's evolution, while, above the critical point, the elastic energy will play a more important role. This can be proved from Fig. 8 (b), in which the simulation time $\left(t^{*}=500\right)$ is near the critical point, as shown in Fig.11 $\left(\mathrm{t}_{\text {critical }}=494\right)$. From that figure (Fig.8 (b)), it can be seen that the particle is not a cuboidal structure but a structure with round corner particles, which means that, at this time, the interface energy may act as a main factor. However, when the simulation time greatly exceeds this point, such as in Fig.8(c), the structure is cuboidal and in alignment, which results from the elastic inhomogeneity and the existence of domains, as discussed earlier.

\subsection{Microstructure morphology of Ni-Al binary alloy for different Al content}

The microstructure of Ni-Al binary alloy with a different $\mathrm{Al}$ content (from 15 at. $\%$ to 20 at.\%) has been simulated. The volume fraction of the $\gamma^{\prime}$ phase changed from $28 \%$ to $73 \%$. The comparison of the morphology of the $\gamma^{\prime}$ precipitate at the late stage of coarsening for a different $\mathrm{Al}$ content is shown in Fig. 10. The reduced simulation time for all cases is the same $\left(t^{*}=2000\right)$. It is shown that the precipitate morphology changes from cuboidal to complexly faceted shapes with the increasing of the volume fraction of the $\gamma^{\prime}$ phase. In the case of small volume 

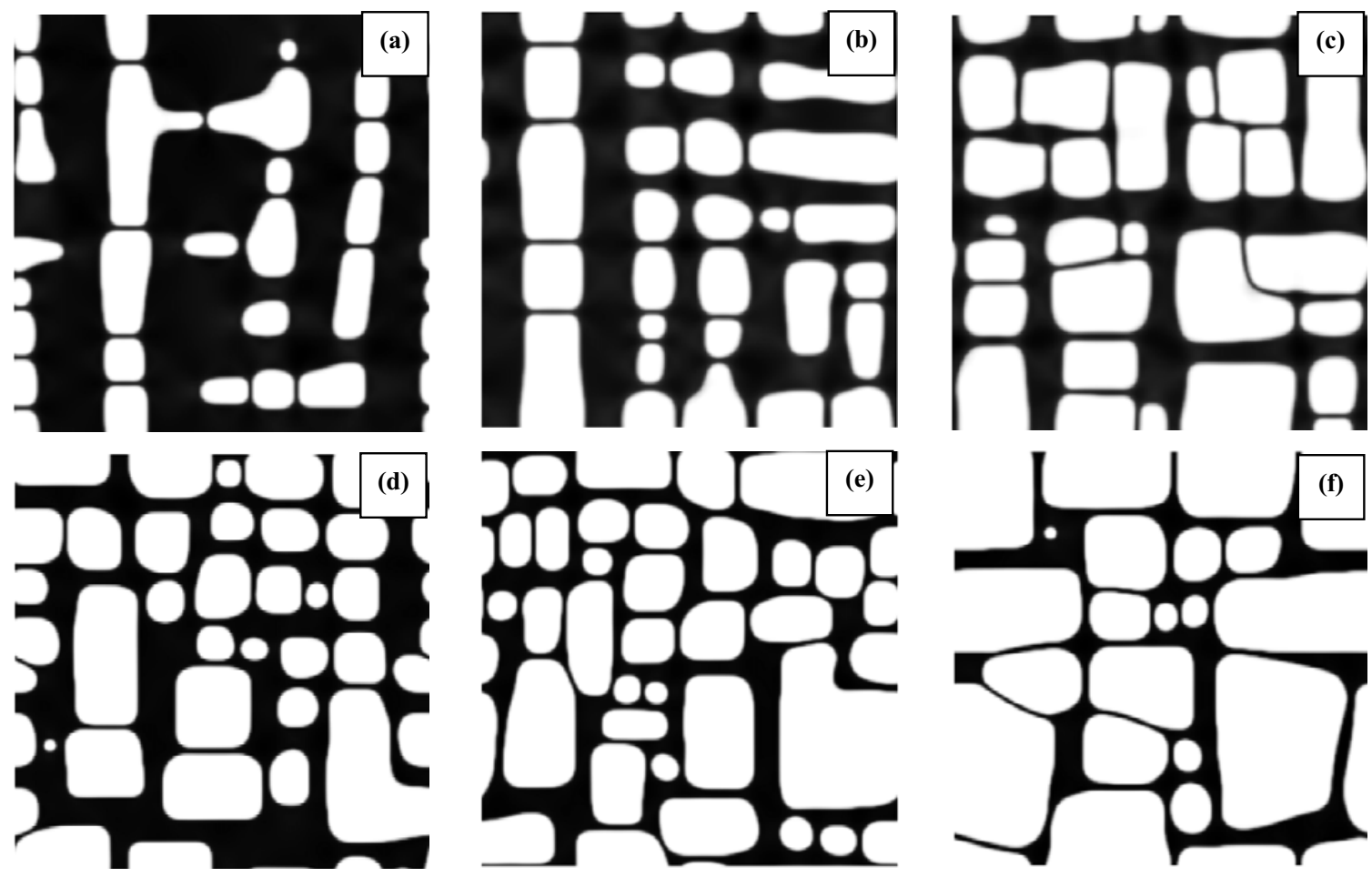

Fig.11 Comparison of the morphology of the $\gamma^{\prime}$ precipitate from the late stages of coarsening for different $\mathrm{Al}$ content. (a) $\mathrm{c}_{\mathrm{Al}}=15 \mathrm{at} . \%$, (b) $\mathrm{c}_{\mathrm{Al}}=16 \mathrm{at} . \%$, (c) $\mathrm{c}_{\mathrm{Al}}=17 \mathrm{at} . \%$, (d) $\mathrm{c}_{\mathrm{Al}}=18 \mathrm{at} . \%$, (e) $\mathrm{c}_{\mathrm{Al}}=19 \mathrm{at} . \%$, (f) $\mathrm{c}_{\mathrm{Al}}=20 \mathrm{at} . \%$
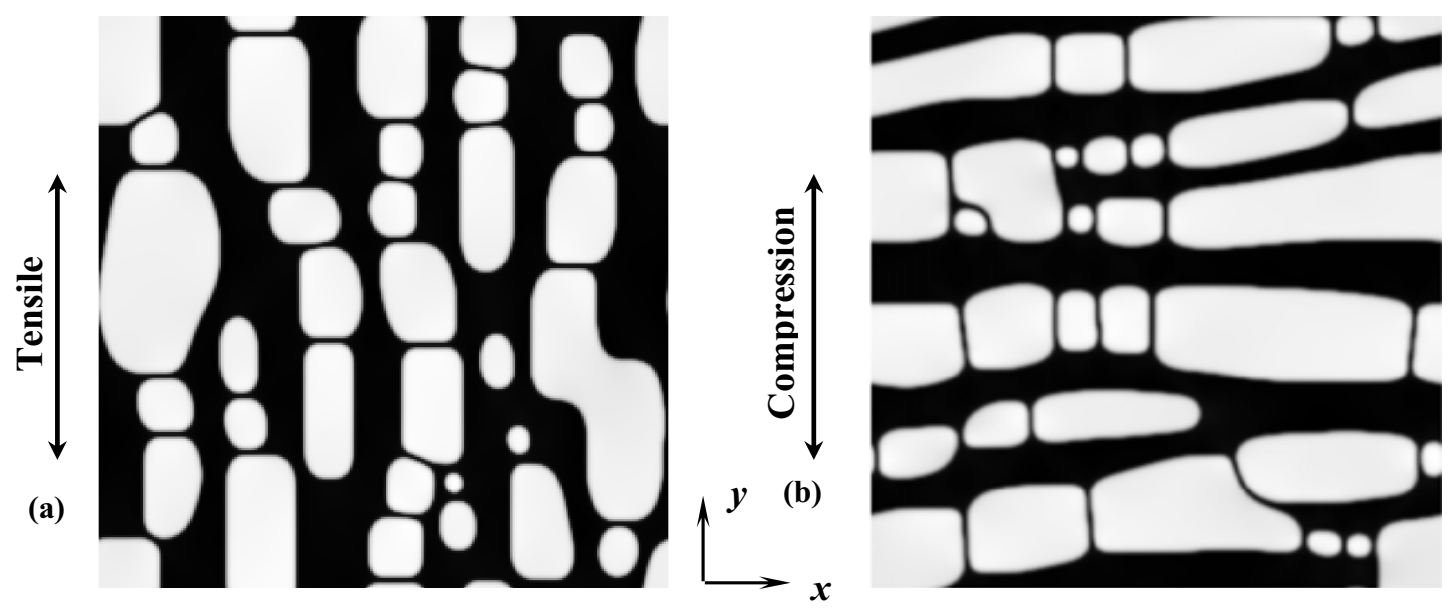

Fig.12 Directional coarsening phenomenon of Ni-Al alloy simulated by PFM with the external strain $\varepsilon_{0}=0.02$ at the reduced simulation time $t^{*}=2000$

(a) Tensile,

(b) Compression

fraction of $\gamma^{\prime}$ precipitate (Fig.11 (a) (c)), the array structures is arranged very regular, however, this array structures break down when the volume fraction is large (Fig.11 (d) (f)). This breakdown of the array structure due to inter-array coalescence leads to complexly faceted precipitate shapes for the large volume fraction. Furthermore, with the increasing of the $\mathrm{Al}$ content in the
Ni-Al binary alloy, the average size of the $\gamma^{\prime}$ particle increases, the size distribution becomes broader, and the aspect ratio of the cuboidal coherent precipitates becomes smaller, which may result from the interactions between the precipitates that become stronger because of the high volume fraction. In addition, because of this interaction, the time for the low-volume fraction 
system to arrive at the equilibrium state is much shorter than that for the system with a high volume fraction.

\subsection{Directional coarsening}

The phenomenon of directional coarsening under the external stress, known as rafting, is of importance, since it is usually observed to take place during service in single crystal superalloys turbine blades of aircraft engines. Experiments have shown that the external stresses can significantly change the morphology of the precipitates and their spatial arrangement. In the present study, the directional coarsening phenomenon of Ni-base superalloy also is simulated by phase field method under this strategy. Fig.11 (a) and (b) show the different microstructures of $\gamma$ and $\gamma^{\prime}$ phases when the external strain are tensile and compression, respectively. The composition is $\mathrm{c}_{\mathrm{Al}}=18 \mathrm{at} . \%$, the applied external strain is $\varepsilon_{0}=0.02$, and the reduced simulation time for both the cases is the same, which is $t^{*}=2000$. The external stress is applied in the y direction as shown in Fig.12. It can be seen that, when the external strain is applied, the microstructure of Ni-base superalloy will change dramatically. The precipitates will change from cuboidal structure plate structure and will align in a certain direction. In the case of tensile strain the $\gamma^{\prime}$ phase will be alignment in the parallel direction of external strain ( $y$ direction), while, when the strain is compressive strain, the coarsening direction is not parallel but normal to the direction of applied external strain ( $x$ direction). The simulation results are accorded with experimental results [26] the Pineatu's [27] directional coarsening map (the lattice misfit of Nial binary alloy is positive). The difference of the elongation direction between tensile case and compression case can be explained by the interaction between external strain and the internal strain caused by the lattice misfit and elastic inhomogeneity between the order and disorder phases. When external strain is tensile, the total strain in the y direction is strengthening, which will make the precipitates elongate in the $y$ direction, while, when it is the case of compression, the strain in the $\mathrm{y}$ direction is decreasing and the strain in the $\mathrm{x}$ direction will increase, which will result the elongation of the precipitates in the $\mathrm{x}$ direction [25]. However, it should be noted that the above explanation is on the base of the order phase is harder that the disorder phase. If the harder phase is the disorder phase, the conclusion will inverse.

\section{Conclusions}

A new strategy for the simulation of microstructure evolution of Ni-base superalloys is proposed by coupling a phase field method with CALPHAD and CVM. Under such a strategy, the microstructure evolution of a $\mathrm{Ni}-\mathrm{Al}$ binary alloy has been simulated, and the elastic energy due to the lattice misfit and different elastic modulus between $\gamma$ phase and $\gamma^{\prime}$ phase also has been considered. The simulated microstructures are formed by a cuboidal coherent $\gamma^{\prime}$ phase with strong alignment in a certain direction. All the precipitates are single-domain particles. The influence of the volume fraction of the $\gamma^{\prime}$ phase on the final microstructures has also been investigated. It is shown that, with the increasing of the volume fraction, the size distribution of the precipitates becomes broader, and the aspect ratio of the cuboidal coherent precipitates becomes smaller, which may result from the interactions between the precipitates that become stronger because of the high volume fraction. Moreover, the directional coarsening phenomenon of $\mathrm{Ni}-\mathrm{Al}$ alloy with 18 at. \%Al has been reoccurred with this strategy when the various external stress conditions are applied, which shows the same conclusion as Pineatu's directional coarsening map. The simulation results demonstrate that the strategy of combining PFM, CALPHAD, and CVM can describe all the complexities involved in the coarsening of the $\gamma^{\prime}$ phase in a Ni-base superalloy.

\section{References}

1. Y. Wang, D. Banerjee, C. C. Su, A. G. Khachaturyan, Acta Mater., 46(1998), 2983-3001.

2. G. Rubin, A. G. Khachaturyan, Acta Mater., 47(1999), 1995-2002.

3. D. Banjerjee, R. Banerjee, Y. Wang, Scripta Materialia, 41(1999), 1023-1030.

4. L. Q. Chen, Y. Z. Wang, JOM, 48(1996), 13-16.

5. V. Vaithyanathan, L. Q. Chen, Acta Mater., 50(2002), 4061-4073.

6. J. Z. Zhu, Z. K. Liu, V. Vaithyanathan, L. Q. Chen, Scripta Materialia, 46(2002), 401-406.

7. U. Grafe, B. Bottger, J. Tiaden, S. G. Fries, Scripta Materialia, 42(2000), 1179-1186.

8. Q. Chen, N. Ma, K. Wu, Y. Wang, Scripta Materialia, 50(2004), 471-476.

9. J. W. Cahn, J. E. Hilliard, J. Chem. Phys., 28(1958), 258.

10. S. Y. Hu, L. Q. Chen, Acta Mater. 49(2001), 1879-1890

11. I. Ansara, N. Dupin, H. L. Lukas, B. Sundman, Journal of Alloys and Compounds, 247(1997), 20-30.

12. M. Enomoto, H. Harada, Metallurgical Transactions A, 20A(1989), 649-664.

13. R. Kikuchi, J. W. Cahn, Acta Metall., 27(1977), 1337.

14. M. Asta, J. J. Hoyt, Acta Mater., 48 (2000), 1089-1096

15. M. Asta, Acta Mater., 44(1996), 4131-4136.

16. A. J. Ardell, Modeling Simul. Mater. Sci. Eng., 8 (2000), 277-286

17. T. Yokokawa, M. Osawa, T. Murakumo, Y. Koizumi, T Kobayashi, H. Harada, $2^{\text {nd }}$ International Symposium, "High-temperature Materials 2001," 2001, 5.31-6.2, Japan, 16-17.

18. A. B. Kamara, A. J. Ardell, C. N. J. Wagner, Metallurgical and Materials Transactions A, 27A(1996), 2888-2896

19. A. J. Ardell, Acta Mater., 20(1972), 61.

20. A. J. Ardell, Interface Science, 3(1995), 119-125.

21. L. Q. Chen, J. Shen, Computer Physics Communications, 108(1998), 147-158.

22. J. Zhu, L. Q. Chen, Physical Review E, 60(1999), 35643572.

23. M. Osawa, H. Harada, $2^{\text {nd }}$ International Symposium, "High-temperature Materials 2001," 2001, 5.31-6.2, Japan, 14-15.

24. H. Harada, A. Ishida, Y. Murakami, H. K. D. H. Bhadeshia, M. Yamazaki, Applied Surface Science, 67(1993), 209 304.

25. D. Y. Li, L. Q. Chen, Scripta Materialia, 37(1997), 1271-1277.

26. F.R.N. Nabarro, Metallurgical and Materials Transactions A, 27A(1996), 513-530

27. A. Pineau, Acta Mater., 24(1976),559. 